\title{
Modulation Transfer Function of a Gaussian Beam Based on the Generalized Modified Atmospheric Spectrum
}

\author{
Chao Gao and Xiaofeng Li \\ School of Astronautics and Aeronautics, University of Electronic Science and Technology of China, 2006 Xiyuan Ave, \\ West Hi-Tech Zone, Chengdu 611731, China \\ Correspondence should be addressed to Xiaofeng Li; lxf3203433@uestc.edu.cn
}

Received 26 May 2016; Accepted 3 August 2016

Academic Editor: Sulaiman Wadi Harun

Copyright (C) 2016 C. Gao and X. Li. This is an open access article distributed under the Creative Commons Attribution License, which permits unrestricted use, distribution, and reproduction in any medium, provided the original work is properly cited.

This paper investigates the modulation transfer function of a Gaussian beam propagating through a horizontal path in weakfluctuation non-Kolmogorov turbulence. Mathematical expressions are obtained based on the generalized modified atmospheric spectrum, which includes the spectral power law value of non-Kolmogorov turbulence, the finite inner and outer scales of turbulence, and other optical parameters of the Gaussian beam. The numerical results indicate that the atmospheric turbulence would produce less negative effects on the wireless optical communication system with an increase in the inner scale of turbulence. Additionally, the increased outer scale of turbulence makes a Gaussian beam influenced more seriously by the atmospheric turbulence.

\section{Introduction}

Optical wireless communication technology has drawn much attention for its significant technological challenges and prospective applications. It uses beams of laser propagating in the atmosphere to wirelessly transmit data at high speed. However, the atmosphere is full of numerous turbulence eddies, which has great degrading impacts on the performance of the communication system. The degrading effects of atmospheric turbulence on the communication system can be characterized statistically by the modulation transfer function (MTF) [1]. In the past few decades, various power spectrum models of refractive index have been proposed to analyze the MTF for different situations. Generally speaking, these turbulence power spectrum models can be classified into two typical categories: Kolmogorov and nonKolmogorov models. The former have a fixed power law value of $11 / 3$, while the latter allow the power law value to vary in the range from three to four. Most non-Kolmogorov models can be generalized from their corresponding Kolmogorov models, and thus the Kolmogorov models can be regarded as specific cases of the non-Kolmogorov models [2]. Among these models, the generalized modified atmospheric spectrum not only considers the variable spectral power law value between the ranges from 3 to 4 , but also takes the finite inner and outer scales of turbulence into account [3]. Besides, the generalized modified atmospheric spectrum features the small rise at a high wavenumber, which is clearly seen in temperature data recorded by sensors. These properties make the generalized modified atmospheric spectrum suitable and unique in the investigation of the MTF for plane and spherical waves [4].

In this study, the generalized modified atmospheric spectrum is used to investigate the MTF of a Gaussian beam in non-Kolmogorov turbulence along a horizontal path. The Gaussian beam, whose transverse electric field and intensity are normally distributed, is a typical kind of electromagnetic wave [5]. The rest of the paper is organized as follows. Section 2 introduces the generalized modified atmospheric spectrum and the MTF of a Gaussian beam. Section 3 presents a detailed expression reduction. The influences of the inner and outer scales of turbulence on the MTF of a Gaussian beam are analyzed in Section 4, followed by conclusions in Section 5.

\section{Theoretical Models}

2.1. Generalized Modified Atmospheric Spectrum. The generalized modified atmospheric spectrum takes the form [3]

$$
\Phi_{n}(\kappa)=A(\alpha) C_{n}^{2} \kappa^{-\alpha} f(\kappa, \alpha),
$$


where $\kappa \in[0,+\infty)$ is the angular wavenumber of the turbulence scale, $\alpha \in(3,4)$ is the spectral power law value, $C_{n}^{2}$ is the generalized atmospheric structure parameter, $l_{0} \geq 0$ is the inner scale of turbulence, and $L_{0} \geq l_{0}$ is the outer scale of turbulence. $A(\alpha)$ in (1) is a function related to $\alpha$ :

$$
A(\alpha)=\frac{\Gamma(\alpha-1)}{4 \pi^{2}} \sin \frac{\pi(\alpha-3)}{2},
$$

where $\Gamma(x)$ is the gamma function.

For the convenience of mathematical analysis, let

$$
\begin{aligned}
C_{\alpha}= & \frac{3-\alpha}{3} \Gamma\left(\frac{3-\alpha}{2}\right)+a \frac{4-\alpha}{3} \Gamma\left(\frac{4-\alpha}{2}\right) \\
& -b \frac{3+\beta-\alpha}{3} \Gamma\left(\frac{3+\beta-\alpha}{2}\right),
\end{aligned}
$$

where the constant coefficients $a, b$, and $\beta$ in (3) are usually set as

$$
\begin{aligned}
& a=1.802, \\
& b=0.254, \\
& \beta=\frac{7}{6} .
\end{aligned}
$$

It must be pointed out that the values of these coefficients are based on the experiments for the classic Kolmogorov turbulence but are widely used for theoretical analyses of non-Kolmogorov turbulence $[1,4]$. Nevertheless, $f(\kappa, \alpha)$ in (1) takes the form

$$
\begin{aligned}
f(\kappa, \alpha)= & \exp \left(-\frac{\kappa^{2}}{\kappa_{l}^{2}}\right) \times\left(1-\exp \left(-\frac{\kappa^{2}}{\kappa_{0}^{2}}\right)\right) \\
& \times\left(1+a\left(\frac{\kappa}{\kappa_{l}}\right)-b\left(\frac{\kappa}{\kappa_{l}}\right)^{\beta}\right) \\
= & \sum_{i=1}^{3} \sum_{j=1}^{2}(-1)^{j-1} c_{i} \kappa^{p_{i}} \exp \left(-d_{j}^{2} \kappa^{2}\right),
\end{aligned}
$$

where

$$
\begin{aligned}
& \kappa_{0}=\frac{4 \pi}{L_{0}}, \\
& \kappa_{l}=\frac{\left(\pi A(\alpha) C_{\alpha}\right)^{1 /(\alpha-5)}}{l_{0}} .
\end{aligned}
$$

And the coefficients are $c_{1}=1, c_{2}=a / \kappa_{l}, c_{3}=-b / \kappa_{l}^{\beta}, p_{1}=0$, $p_{2}=1, p_{3}=\beta, d_{1}=\sqrt{1 / \kappa_{l}^{2}}$, and $d_{2}=\sqrt{1 / \kappa_{l}^{2}+1 / \kappa_{0}^{2}}$.

2.2. MTF of a Gaussian Beam. The MTF is relative to the wave structure function (WSF). Based on the Rytov approximation, the WSF of Gaussian beam takes the simple form [1]

$$
D(\rho)=8 L k^{2} \pi^{2} \int_{0}^{1} \mathrm{~d} \xi \int_{0}^{+\infty} \mathrm{d} \kappa
$$

$$
\begin{aligned}
& \times \kappa \Phi_{n}(\kappa) \exp \left(-\frac{L \Lambda \kappa^{2} \xi^{2}}{k}\right) \\
& \times\left(I_{0}(\Lambda \rho \kappa \xi)-J_{0}(\rho \kappa(1-\bar{\Theta} \xi))\right),
\end{aligned}
$$

where $\rho$ is the scalar separation between two observation points and $L$ is the propagation optical path length. $k$ in (7) is the angular wavenumber of Gaussian beam wave

$$
k=\frac{2 \pi}{\lambda}
$$

where $\lambda$ is the wavelength of Gaussian beam. Both $\Lambda$ and $\bar{\Theta}$ in (7) are optical parameters of the Gaussian beam at the receiver

$$
\begin{aligned}
& \Theta=\frac{\Theta_{0}}{\Theta_{0}^{2}+\Lambda_{0}^{2}}, \\
& \bar{\Theta}=1-\Theta, \\
& \Lambda=\frac{\Lambda_{0}}{\Theta_{0}^{2}+\Lambda_{0}^{2}},
\end{aligned}
$$

where $\Theta_{0}$ is the curvature parameter of Gaussian beam at transmitter and $\Lambda_{0}$ is the Fresnel ratio of Gaussian beam at transmitter

$$
\begin{aligned}
& \Theta_{0}=1-\frac{L}{R_{0}}, \\
& \Lambda_{0}=\frac{2 L}{k W_{0}} .
\end{aligned}
$$

In (10), $R_{0}$ is the phase front radius of Gaussian beam at transmitter, and $W_{0}$ is the radius of Gaussian beam at transmitter. $I_{0}(x)$ in (7) is the modified Bessel function of the first kind with zero order, and $J_{0}(x)$ in (7) is the Bessel function of the first kind with zero order [6]

$$
\begin{aligned}
& I_{0}(x)=\sum_{n=0}^{+\infty} \frac{1}{(n !)^{2}}\left(\frac{x}{2}\right)^{2 n} \\
& J_{0}(x)=\sum_{n=0}^{+\infty} \frac{(-1)^{n}}{(n !)^{2}}\left(\frac{x}{2}\right)^{2 n}
\end{aligned}
$$

The atmospheric turbulence MTF takes the form [1]

$$
\operatorname{MTF}(\mu)=\exp \left(-\frac{1}{2} D(\mu d)\right)
$$

where $\mu$ is the normalized spatial frequency and $d$ is the receiver aperture diameter. It is clear that the value range of the MTF is the interval from 0 to 1.

\section{Expression Reduction}

The calculation equation (7) will spend too much time because of its improper iterated integral. As an alternative, the closed-form expression of (7) can replace the improper iterated integral with special functions, which has corresponding 
packages in frequently used software. This section mainly discusses the reduction of (7).

Substituting (1) into (7), it follows that

$$
\begin{aligned}
D(\rho)= & 8 A(\alpha) C_{n}^{2} L k^{2} \pi^{2} \int_{0}^{1} \mathrm{~d} \xi \int_{0}^{+\infty} \mathrm{d} \kappa \\
& \times \kappa^{1-\alpha} f(\kappa, \alpha) \exp \left(-\frac{L \Lambda \kappa^{2} \xi^{2}}{k}\right) \\
& \times\left(I_{0}(\Lambda \rho \kappa \xi)-J_{0}(\rho \kappa(1-\bar{\Theta} \xi))\right) .
\end{aligned}
$$

For mathematical convenience, let

$$
\begin{aligned}
D_{I}= & \int_{0}^{1} \mathrm{~d} \xi \int_{0}^{+\infty} \mathrm{d} \kappa \times \kappa^{1-\alpha} f(\kappa, \alpha) \exp \left(-\frac{L \Lambda \kappa^{2} \xi^{2}}{k}\right) \\
& \times\left(I_{0}(\Lambda \rho \kappa \xi)-1\right), \\
D_{J}= & \int_{0}^{1} \mathrm{~d} \xi \int_{0}^{+\infty} \mathrm{d} \kappa \times \kappa^{1-\alpha} f(\kappa, \alpha) \exp \left(-\frac{L \Lambda \kappa^{2} \xi^{2}}{k}\right) \\
& \times\left(J_{0}(\rho \kappa(1-\bar{\Theta} \xi))-1\right) .
\end{aligned}
$$

Thus, (13) can be presented by

$$
D(\rho)=8 A(\alpha) C_{n}^{2} L k^{2} \pi^{2} \times\left(D_{I}-D_{J}\right) .
$$

3.1. Reduction of $D_{I}$. Substituting (11) into (14), $D_{I}$ is rewritten as

$$
\begin{aligned}
D_{I}= & \int_{0}^{1} \mathrm{~d} \xi \int_{0}^{+\infty} \mathrm{d} \kappa \times \sum_{n=1}^{+\infty} \frac{1}{(n !)^{2}}\left(\frac{\Lambda \rho \kappa \xi}{2}\right)^{2 n} \\
& \times \kappa^{1-\alpha} f(\kappa, \alpha) \exp \left(-\frac{L \Lambda \kappa^{2} \xi^{2}}{k}\right) .
\end{aligned}
$$

In most situations, $\rho \ll 1$. This is because MTF will quickly converge to zero when $\rho$ approaches one; that is, MTF is significantly larger than zero when $\rho$ approaches zero. Thus, (16) could be approximated by the simpler expression

$$
\begin{aligned}
D_{I} \approx & \frac{\Lambda^{2} \rho^{2}}{4} \int_{0}^{1} \xi^{2} \mathrm{~d} \xi \int_{0}^{+\infty} \mathrm{d} \kappa \\
& \times \kappa^{3-\alpha} f(\kappa, \alpha) \exp \left(-\frac{L \Lambda \kappa^{2} \xi^{2}}{k}\right) .
\end{aligned}
$$

Consider the iterated integral in (17). According to (5), there is

$$
\begin{aligned}
\kappa^{3-\alpha} f(\kappa, \alpha) \exp \left(-\frac{L \Lambda \kappa^{2} \xi^{2}}{k}\right) \\
=\sum_{i=1}^{3} \sum_{j=1}^{2}(-1)^{j-1} c_{i} \kappa^{3-\alpha+p_{i}} \\
\quad \times \exp \left(-\left(d_{j}^{2}+\frac{L \Lambda \xi^{2}}{k}\right) \kappa^{2}\right) .
\end{aligned}
$$

Based on the equation for $u>-1$ and $v>0$ [7],

$$
\int_{0}^{+\infty} x^{u} \exp \left(-v x^{2}\right) \mathrm{d} x=\frac{1}{2} v^{-(u+1) / 2} \Gamma\left(\frac{u+1}{2}\right),
$$

we can get

$$
\begin{aligned}
& \int_{0}^{+\infty} \kappa^{3-\alpha} f(\kappa, \alpha) \exp \left(-\frac{L \Lambda \kappa^{2} \xi^{2}}{k}\right) \mathrm{d} \kappa \\
& =\sum_{i=1}^{3} \sum_{j=1}^{2}(-1)^{j-1} \frac{c_{i}}{2}\left(d_{j}^{2}+\frac{L \Lambda \xi^{2}}{k}\right)^{-\left(4-\alpha+p_{i}\right) / 2} \\
& \quad \times \Gamma\left(\frac{4-\alpha+p_{i}}{2}\right)
\end{aligned}
$$

Without loss of generality, the integrand in (17) takes the form

$$
\begin{aligned}
\xi^{n} \int_{0}^{+\infty} \kappa^{p} f(\kappa, \alpha) \exp \left(-\frac{L \Lambda \kappa^{2} \xi^{2}}{k}\right) \mathrm{d} \kappa \\
\quad=\sum_{i=1}^{3} \sum_{j=1}^{2}(-1)^{j-1} \frac{c_{i}}{2} \Gamma\left(\frac{1+p+p_{i}}{2}\right) \\
\quad \times\left(\frac{L \Lambda}{k}\right)^{-\left(1+p+p_{i}\right) / 2} \xi^{n}\left(\frac{k d_{j}^{2}}{L \Lambda}+\xi^{2}\right)^{-\left(1+p+p_{i}\right) / 2},
\end{aligned}
$$

where $n=2$ and $p=3-\alpha$. Based on the equation for $u>0$ and $w>0$ [7],

$$
\begin{aligned}
\int_{0}^{1} x^{u-1}\left(w^{2}+x^{2}\right)^{v} \mathrm{~d} x \\
\quad=\frac{1}{u} w^{2 v}{ }_{2} F_{1}\left(-v, \frac{u}{2} ; \frac{u+2}{2} ;-\frac{1}{w^{2}}\right),
\end{aligned}
$$

we can get

$$
\begin{gathered}
\int_{0}^{1} \xi^{n} \mathrm{~d} \xi \int_{0}^{+\infty} \mathrm{d} \kappa \times \kappa^{p} f(\kappa, \alpha) \exp \left(-\frac{L \Lambda \kappa^{2} \xi^{2}}{k}\right) \\
=\sum_{i=1}^{3} \sum_{j=1}^{2}(-1)^{j-1} \frac{c_{i}}{2 d_{j}^{1+p+p_{i}}(n+1)} \times \Gamma\left(\frac{1+p+p_{i}}{2}\right) \\
\quad \times{ }_{2} F_{1}\left(\frac{1+p+p_{i}}{2}, \frac{n+1}{2} ; \frac{n+3}{2} ;-\frac{L \Lambda}{k d_{j}^{2}}\right)
\end{gathered}
$$

where ${ }_{2} F_{1}(a, b ; c ; z)$ is the Gaussian hypergeometric function [6]. Thus, $D_{I}$ can be computed by (17) and (23) with $n=2$.

3.2. Reduction of $D_{I}$. Following similar procedures as presented in Section 3.1, $D_{J}$ in (14) is rewritten as

$$
\begin{aligned}
D_{J} \approx & -\frac{\rho^{2}}{4} \int_{0}^{1}(1-\bar{\Theta} \xi)^{2} \mathrm{~d} \xi \int_{0}^{+\infty} \mathrm{d} \kappa \\
& \times \kappa^{3-\alpha} f(\kappa, \alpha) \exp \left(-\frac{L \Lambda \kappa^{2} \xi^{2}}{k}\right) .
\end{aligned}
$$



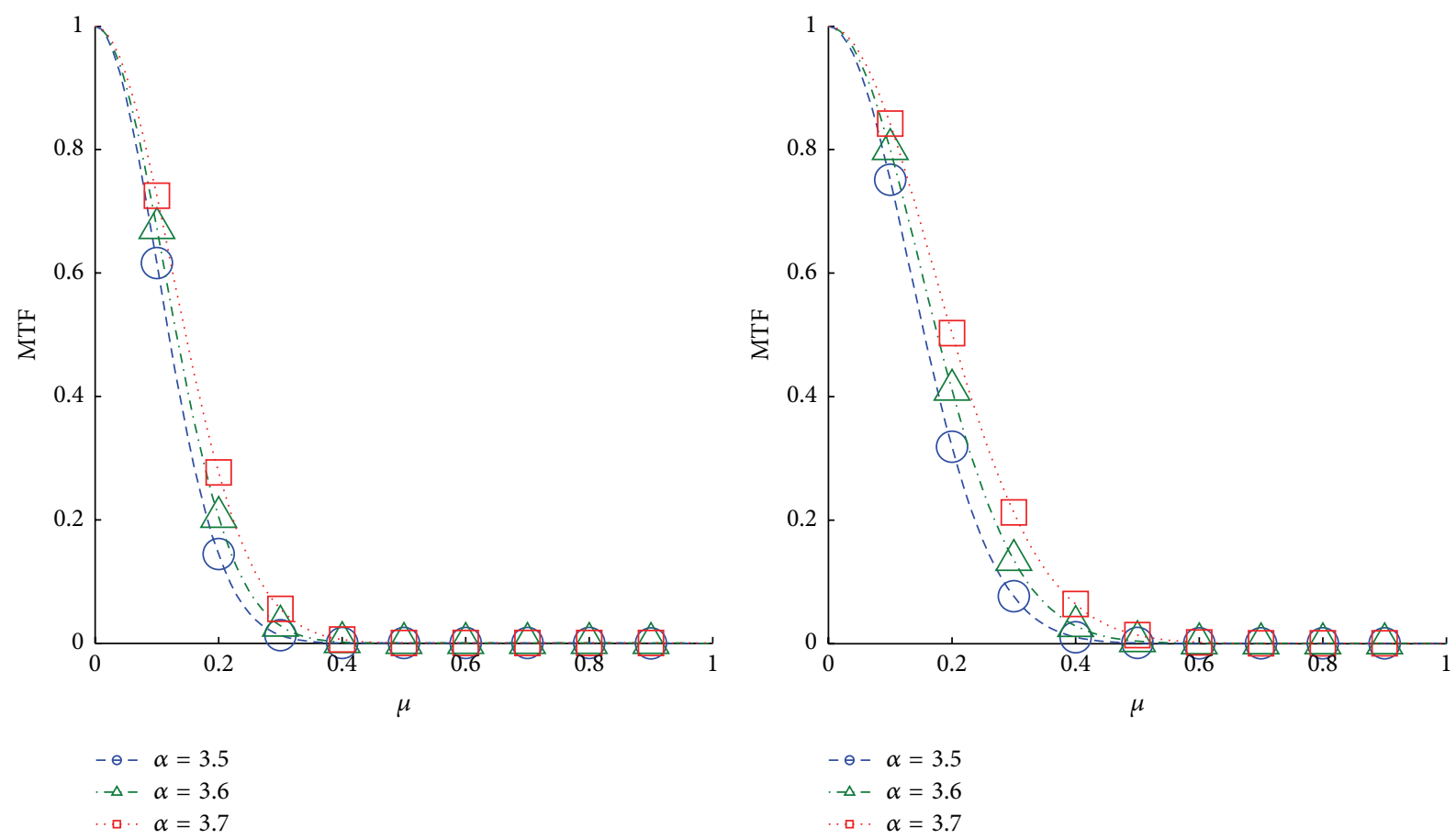

(a) Convergent $\Theta_{0}=0.5$

(b) Collimated $\Theta_{0}=1$

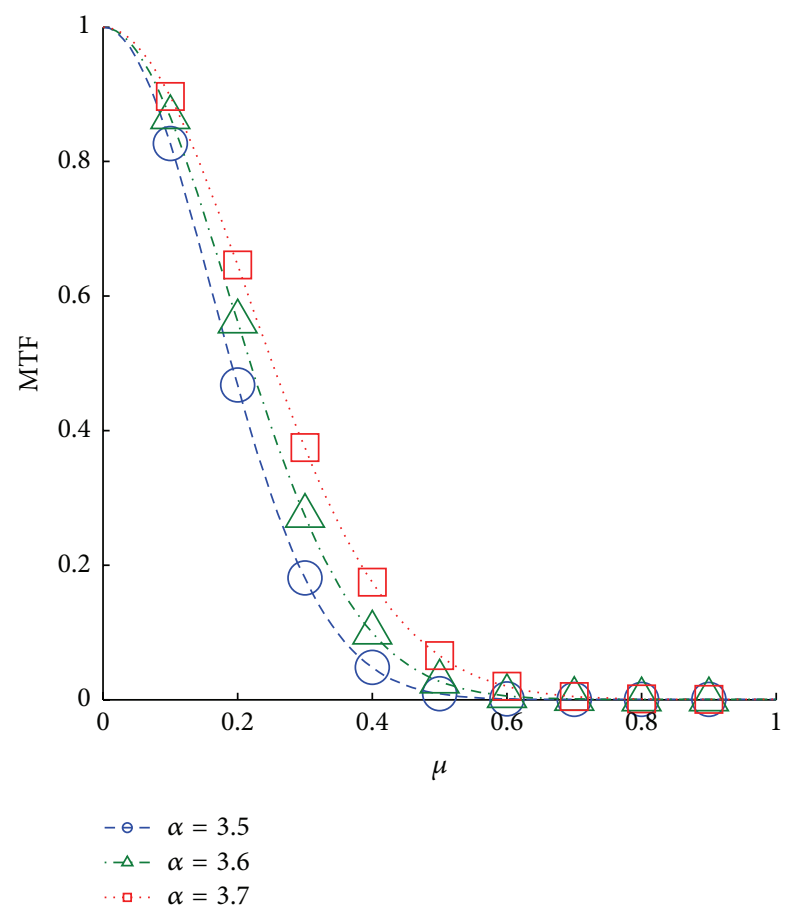

(c) Divergent $\Theta_{0}=2$

FigURE 1: Effects of spectral power law value on MTF for different types of Gaussian beams.

Expanding (24) by the binomial theorem, it follows that

$$
\begin{aligned}
D_{J}= & -\frac{\bar{\Theta}^{2} \rho^{2}}{4} \int_{0}^{1} \xi^{2} \mathrm{~d} \xi \int_{0}^{+\infty} \mathrm{d} \kappa \\
& \times \kappa^{3-\alpha} f(\kappa, \alpha) \exp \left(-\frac{L \Lambda \kappa^{2} \xi^{2}}{k}\right)
\end{aligned}
$$

$$
\begin{aligned}
& +\frac{\bar{\Theta} \rho^{2}}{2} \int_{0}^{1} \xi \mathrm{d} \xi \int_{0}^{+\infty} \mathrm{d} \kappa \\
& \times \kappa^{3-\alpha} f(\kappa, \alpha) \exp \left(-\frac{L \Lambda \kappa^{2} \xi^{2}}{k}\right) \\
& -\frac{\rho^{2}}{4} \int_{0}^{1} \mathrm{~d} \xi \int_{0}^{+\infty} \mathrm{d} \kappa
\end{aligned}
$$




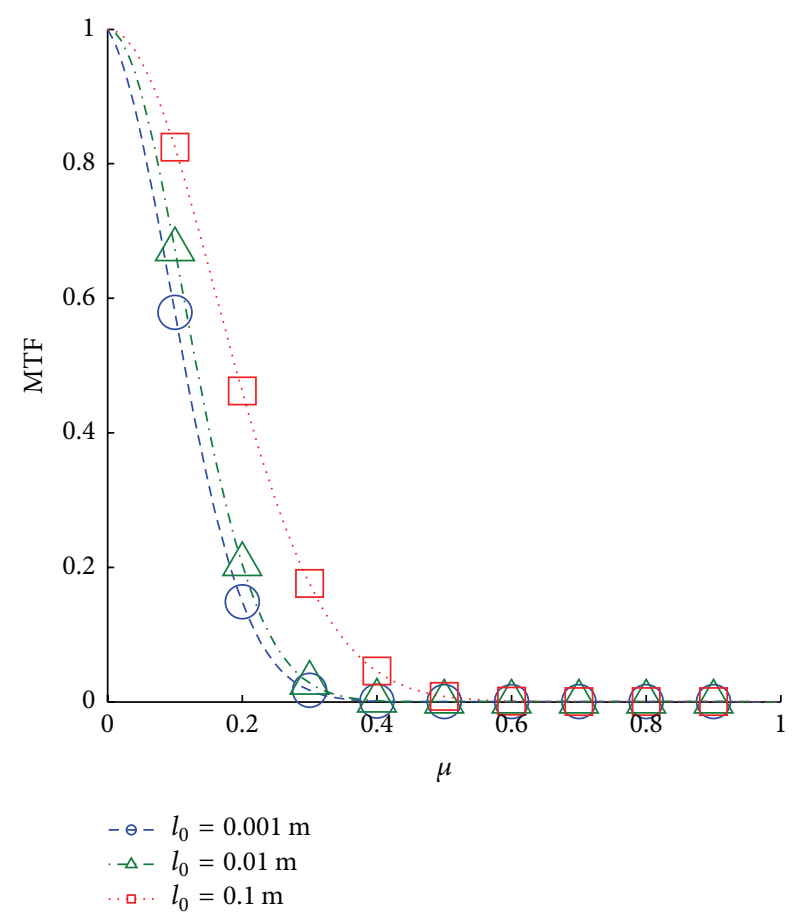

(a) Convergent $\Theta_{0}=0.5$

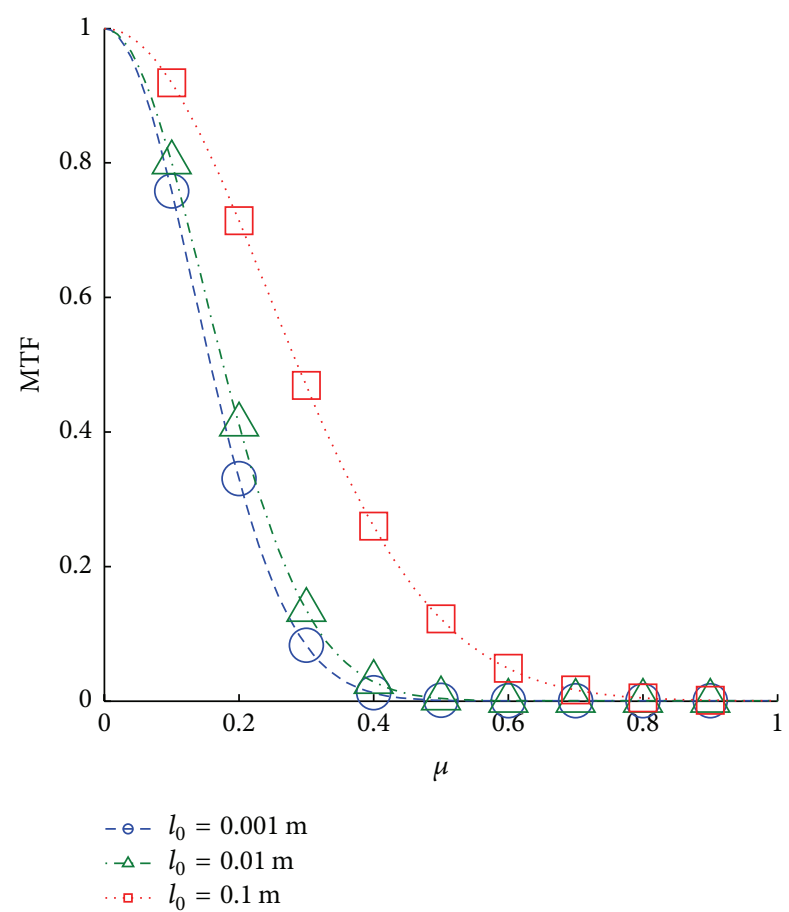

(b) Collimated $\Theta_{0}=1$

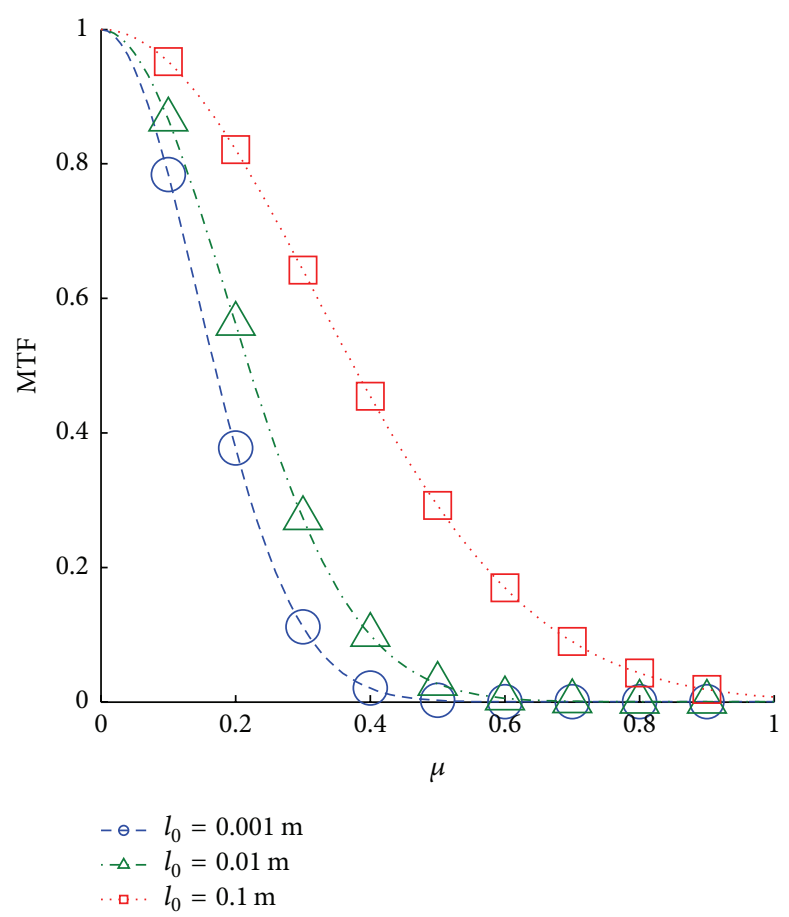

(c) Divergent $\Theta_{0}=2$

FIGURE 2: Effects of inner scale on MTF for different types of Gaussian beams.

$$
\times \kappa^{3-\alpha} f(\kappa, \alpha) \exp \left(-\frac{L \Lambda \kappa^{2} \xi^{2}}{k}\right)
$$

Thus, $D_{J}$ could be computed by (25) and (23) with $n=0,1,2$.

\section{Numerical Simulations}

The following simulations are conducted by the Gaussian beam with these settings: $\lambda=1.55 \times 10^{-6} \mathrm{~m}, L=1000 \mathrm{~m}$, $k \approx 4.0537 \times 10^{6} \mathrm{rad} / \mathrm{m}, C_{n}^{2}=1.7 \times 10^{-14} \mathrm{~m}^{3-\alpha}, W_{0}=0.1 \mathrm{~m}$, 


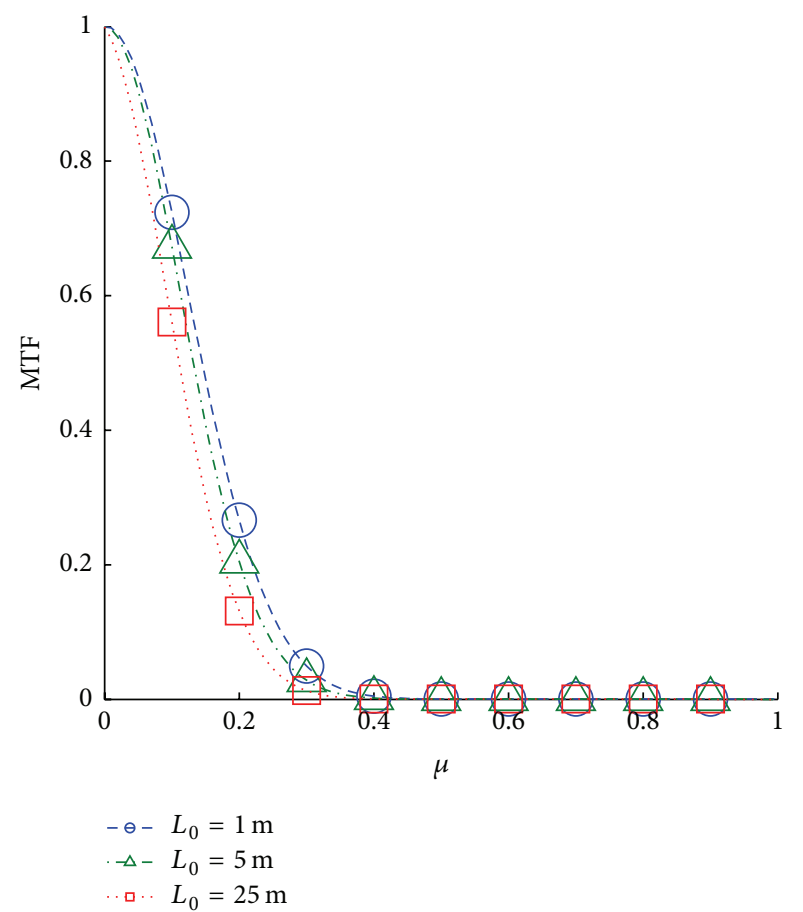

(a) Convergent $\Theta_{0}=0.5$

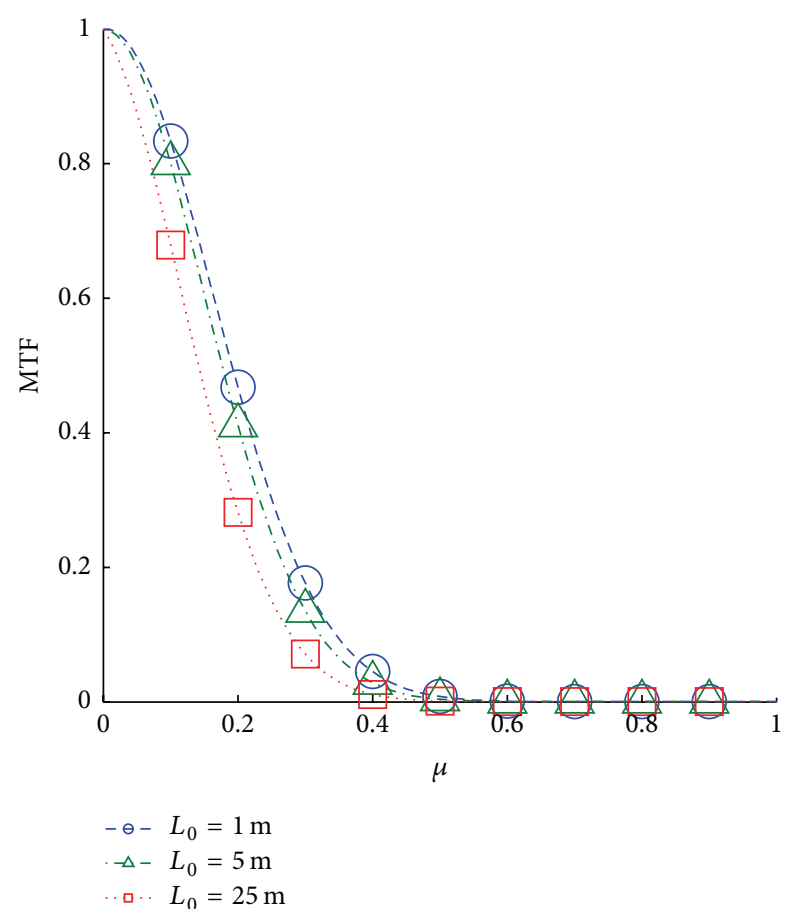

(b) Collimated $\Theta_{0}=1$

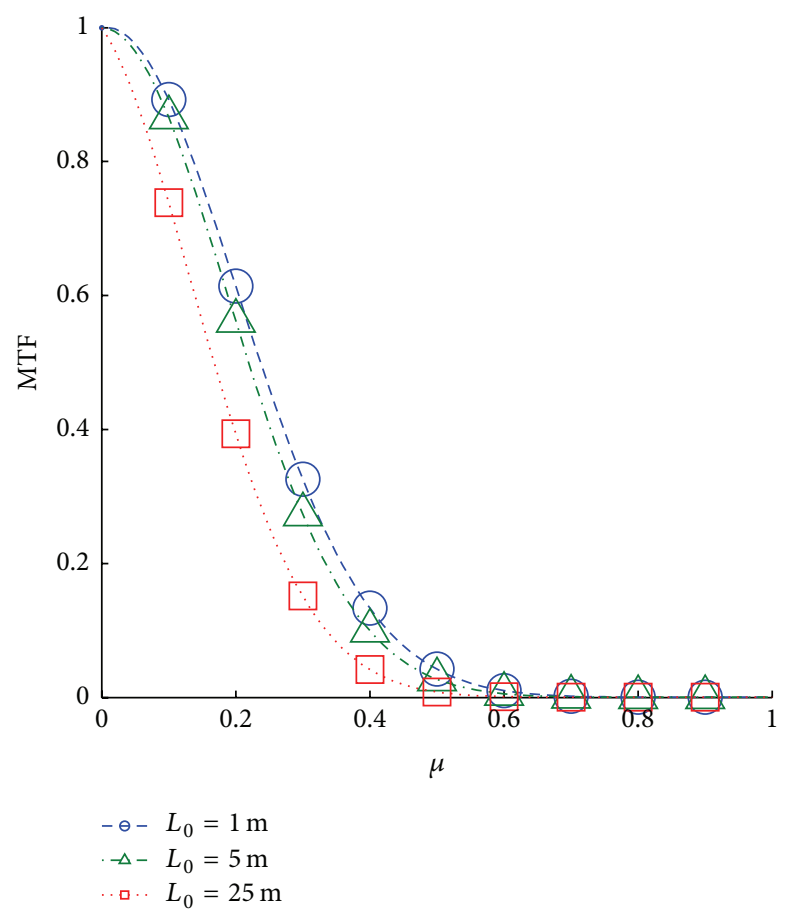

(c) Divergent $\Theta_{0}=2$

FIgURE 3: Effects of outer scale on MTF for different types of Gaussian beams.

$\Lambda_{0} \approx 0.0493$, and $d=0.1 \mathrm{~m}$. Of course, other values can also be chosen.

Figure 1 depicts the effects of spectral power law value on MTF for different types of Gaussian beams. In this calculation, the inner and outer scales of turbulence are set as $l_{0}=0.01 \mathrm{~m}$ and $L_{0}=5 \mathrm{~m}$, respectively. As shown in Figure 1(a), the atmospheric turbulence apparently produces more effects on the propagation of the convergent Gaussian beam $\left(\Theta_{0}=0.5\right)$ with an increase in the normalized spatial frequency $\mu$, which acts in accordance with common sense. Besides, from Figure 1(a), it can be found that the non-Kolmogorov atmospheric turbulence would bring more effects on the wireless optical communication system when the spectral power law value $\alpha$ decreases. The same trends 


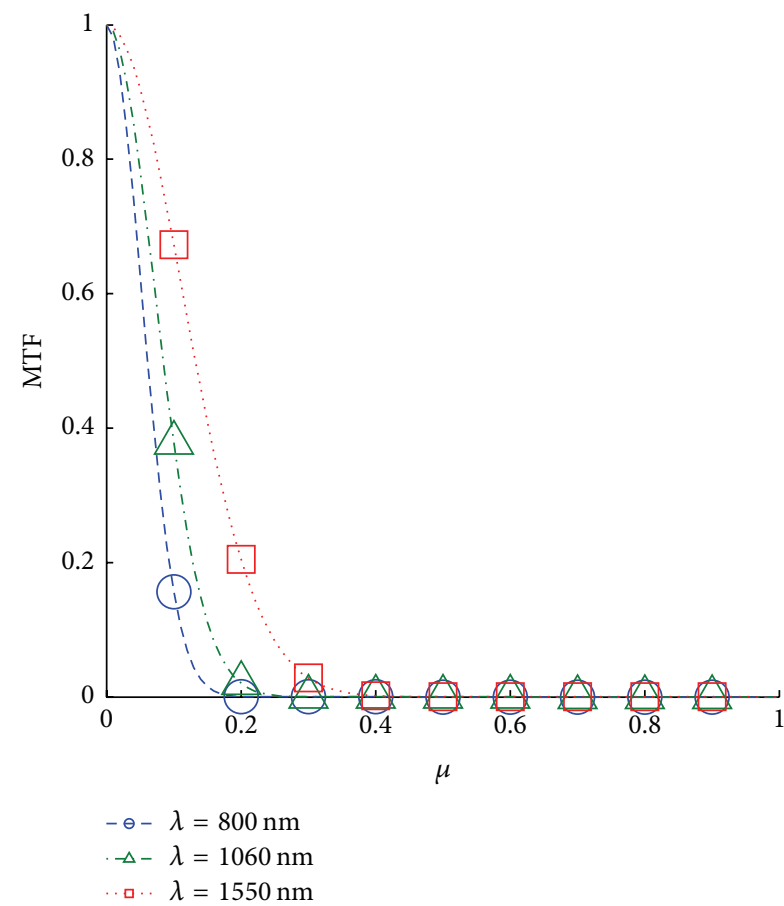

(a) Convergent $\Theta_{0}=0.5$

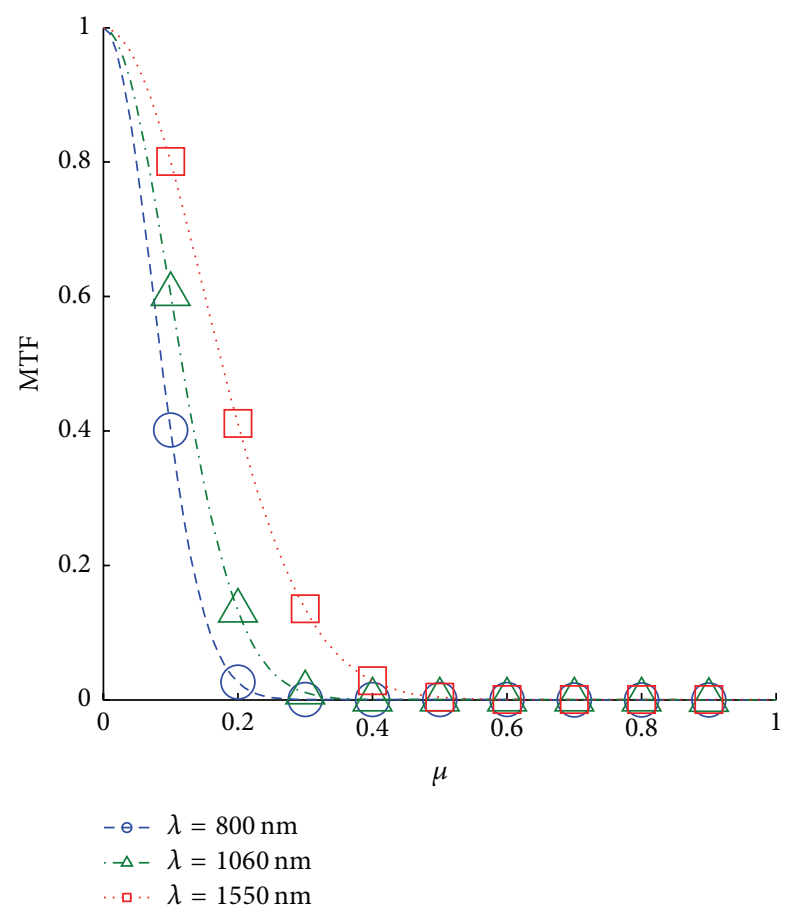

(b) Collimated $\Theta_{0}=1$

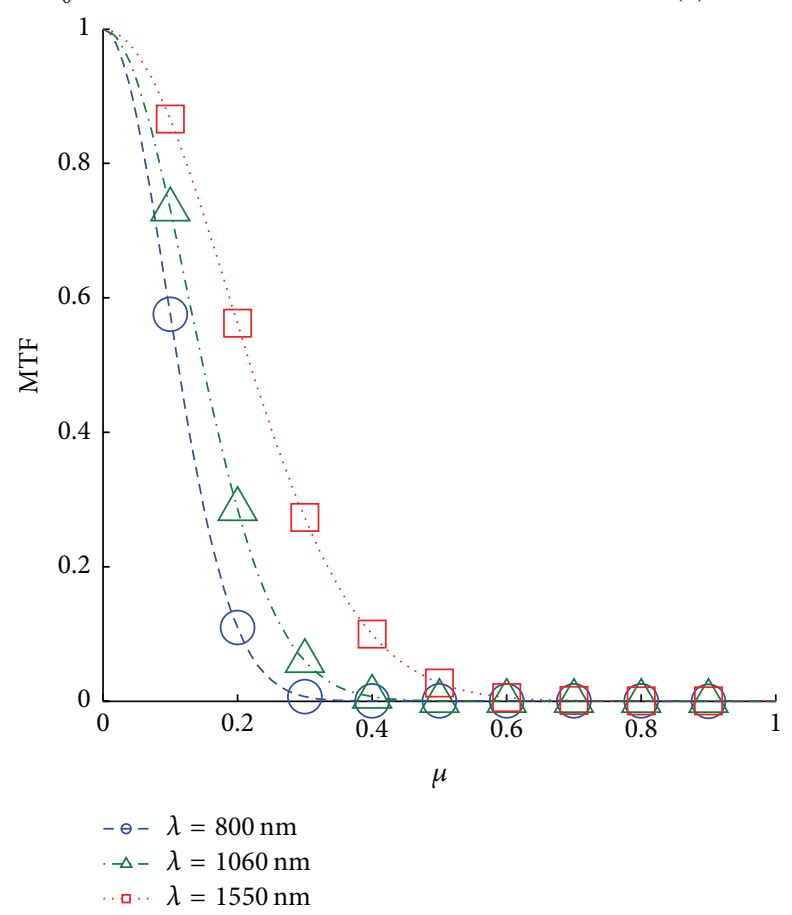

(c) Divergent $\Theta_{0}=2$

FIGURE 4: Effects of wavelength on MTF for different types of Gaussian beams.

are obtained for the collimated Gaussian beam $\left(\Theta_{0}=1\right)$ in Figure $1(\mathrm{~b})$ and the divergent Gaussian beam $\left(\Theta_{0}=2\right)$ in Figure 1(c).

To analyze the effects of the turbulence inner scale on MTF, the spectral power law value and the outer scale of turbulence are fixed to constant values as $\alpha=3.6$ and $L_{0}=5 \mathrm{~m}$. Several inner scales of turbulence are used, and calculation results are depicted in Figure 2 for different types of Gaussian beams. It can be seen that, with an increase in the inner scale of turbulence, the value of MTF also increases. This can be physically explained by the change of inertial subrange of turbulence. When the inner scale of turbulence increases, the frequency's upper bound of inertial subrange would move to a lower position, and thus the atmospheric turbulence would bring less effects on the propagation of the Gaussian beam. 
The influences of outer scale of turbulence on MTF are depicted in Figure 3 for different types of Gaussian beams. For the real atmospheric turbulence, the outer scale of turbulence is usually in the order of meters. Hence, it is set to $1 \mathrm{~m}, 5 \mathrm{~m}$, and $25 \mathrm{~m}$, respectively. The spectral power law value and the inner scale of turbulence are set to $\alpha=3.6$ and $l_{0}=0.01 \mathrm{~m}$ as example. It can be seen that, with an increase in the outer scale of turbulence, the value of MTF decreases and thus the quality of the Gaussian beam is degraded severely by the atmospheric turbulence. This is because WSF is mostly influenced by the large-scale turbulence eddies, which are relevant to the lowfrequency part of the atmospheric turbulence spectrum. A larger turbulence outer scale would lead to a larger range of inertial subrange.

For further discussions and analyses, the inner and outer scales of turbulence are assigned to constant values $l_{0}=$ $0.01 \mathrm{~m}$ and $L_{0}=5 \mathrm{~m}$, respectively. The spectral power law value still uses the default value $\alpha=3$.6. Some typical values of wavelength in the near infrared region, $\lambda=850 \mathrm{~nm}$, $\lambda=1060 \mathrm{~nm}$, and $\lambda=1550 \mathrm{~nm}$, are investigated in this simulation. Figure 4 depicts MTF for different Gaussian beams as a function of $\mu$ with different $\lambda$. It is obvious that the value of MTF increases with an increase in $\lambda$ for certain type of Gaussian beam if other optical parameters are fixed. This phenomenon may be caused by the fact that the larger the beam wavelength, the more pronounced the diffraction. Thus, a laser beam with larger wavelength can be less affected by turbulence eddies.

\section{Conclusions}

In this paper, a theoretical expression of the MTF is derived for a Gaussian beam propagating through the nonKolmogorov atmospheric turbulence along a horizontal path. This expression contains a variable spectral power law value, finite inner and outer scales of turbulence, and other important optical parameters of a Gaussian beam. Numerical simulations indicate that the atmospheric turbulence would produce less degrading effects on the wireless optical communication system with an increase in the spectral power law value. The decreased inner scale of turbulence makes a Gaussian beam influenced more seriously by the atmospheric turbulence. With an increase in the outer scale of turbulence, the quality of a Gaussian beam is degraded more severely by the atmospheric turbulence. A laser beam with larger wavelength can be less affected by turbulence eddies.

\section{Competing Interests}

The authors declare that there is no conflict of interests regarding the publication of this paper.

\section{References}

[1] L. C. Andrews and R. L. Phillips, Laser-Beam Propagation through Random Media, SPIE Optical Engineering Press, Bellingham, Wash, USA, 2nd edition, 2005.

[2] C. Gao, Y. Li, Y. Li, and X. Li, "Irradiance scintillation index for a gaussian beam based on the generalized modified atmospheric spectrum with aperture averaged," International Journal of Optics, vol. 2016, Article ID 8730609, 8 pages, 2016.

[3] B. Xue, L. Cui, W. Xue, X. Bai, and F. Zhou, "Generalized modified atmospheric spectral model for optical wave propagating through non-Kolmogorov turbulence," Journal of the Optical Society of America A: Optics, Image Science, and Vision, vol. 28, no. 5, pp. 912-916, 2011.

[4] B. Xue, L. Cao, L. Cui, X. Bai, X. Cao, and F. Zhou, "Analysis of non-Kolmogorov weak turbulence effects on infrared imaging by atmospheric turbulence MTF," Optics Communications, vol. 300, no. 1, pp. 114-118, 2013.

[5] C. Gao and X. Li, "An analytic expression for the beam wander of a Gaussian wave propagating through scale-dependent anisotropic turbulence," Iranian Journal of Science and Technology, Transactions A: Science, 2016.

[6] F. W. J. Olver, D. W. Lozier, R. F. Boisvert, and C. W. Clark, NIST Handbook of Mathematical Functions, Cambridge University Press, New York, NY, USA, 2010.

[7] I. S. Gradshteyn and I. M. Ryzhik, Table of Integrals, Series, and Products, Academic Press, Waltham, Mass, USA, 8th edition, 2014. 

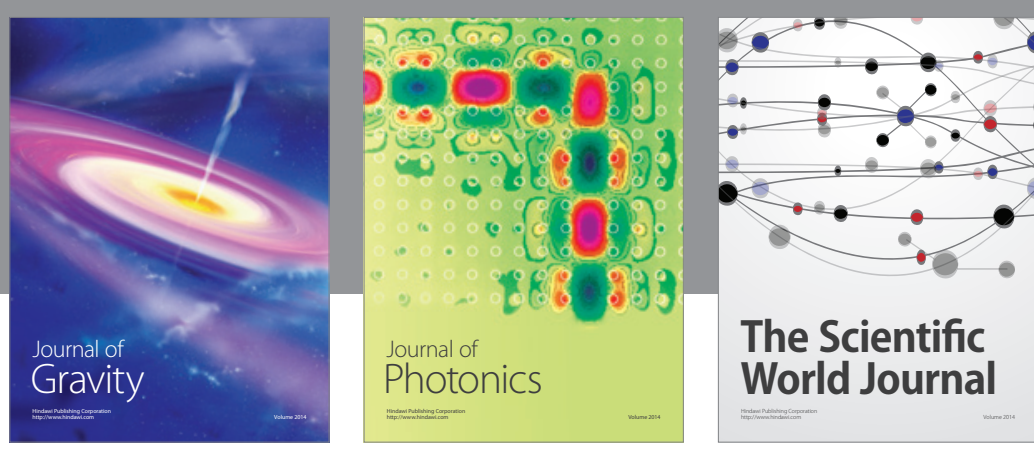

The Scientific World Journal
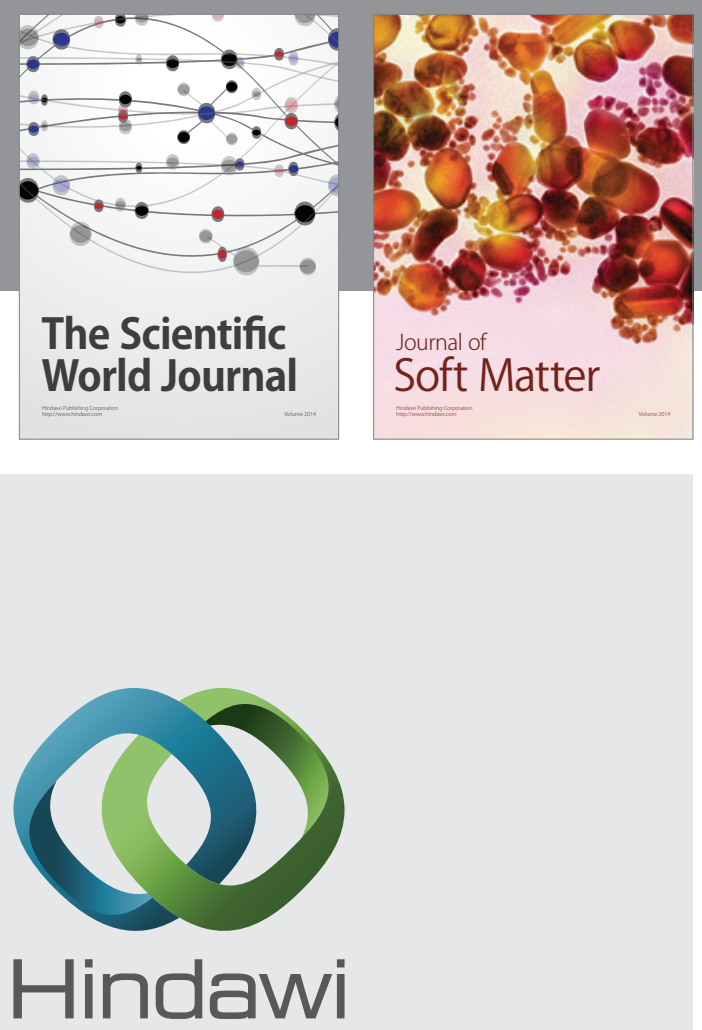

Submit your manuscripts at

http://www.hindawi.com

nternational Journal of

Statistical Mechanics
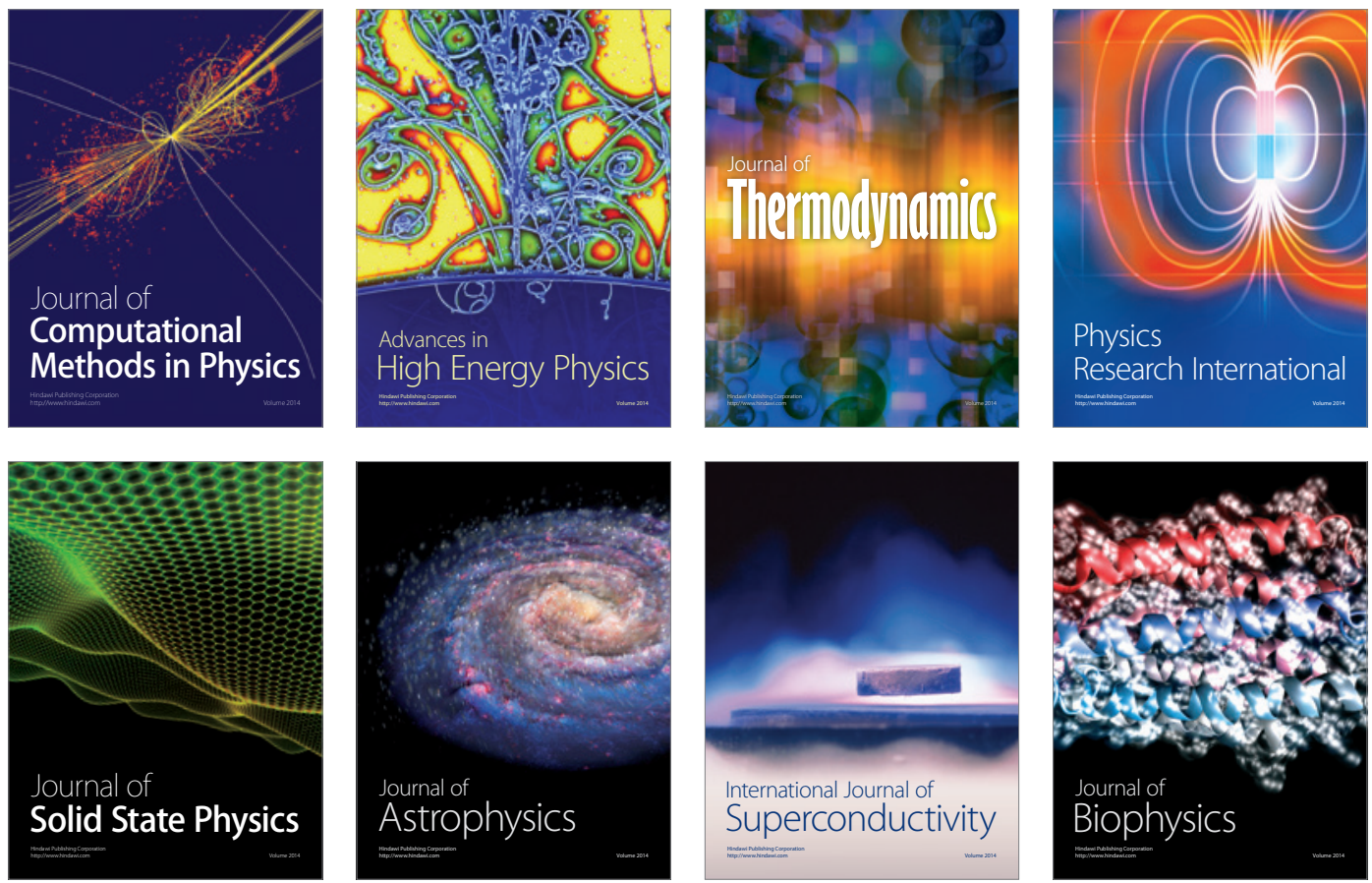
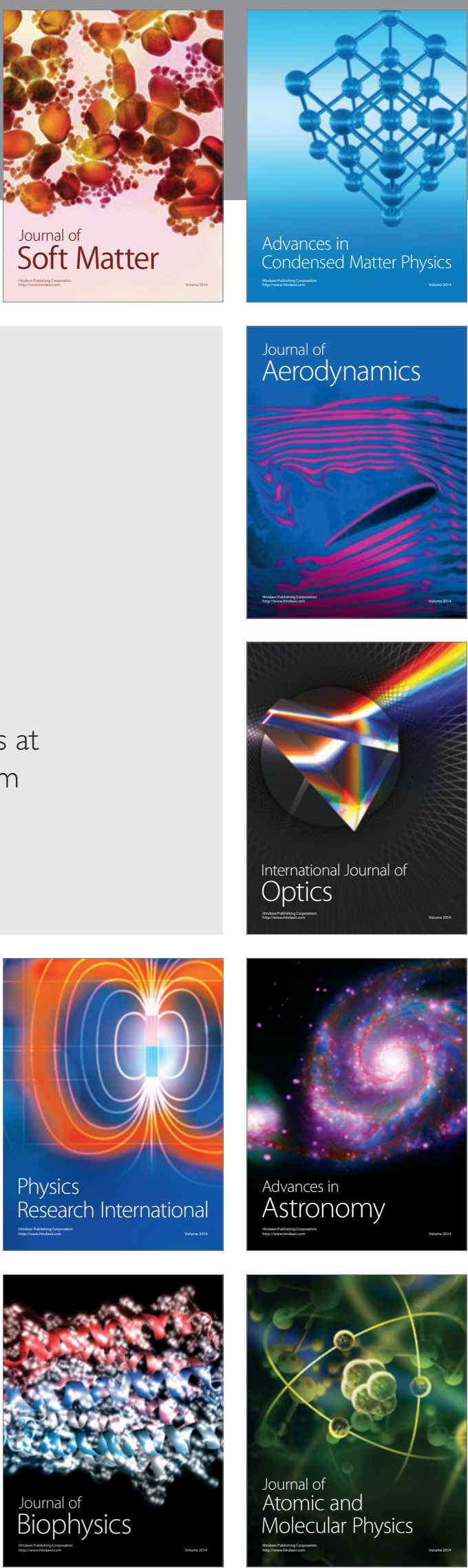